\title{
Performance of Convolution AND CRC CHANNEL ENCODED V-BLAST 4×4 MIMO MC- CDMA WIRELESS COMMUNICATION SYSTEM
}

\author{
Md. Firoz Ahmed ${ }^{1}$, Md. Tahidul Islam ${ }^{1}$ and Abu Zafor Md. Touhidul Islam² \\ 1Department of Information and Communication Engineering, University \\ of Rajshahi, Rajshahi 6205, Bangladesh \\ 2Department of Electrical and Electronic Engineering, University \\ of Rajshahi, Rajshahi 6205, Bangladesh
}

\begin{abstract}
Wireless communications are among the rapidly growing fields in our current life and have a massive effect on every aspect of our everyday life. In this paper, the performance of the various digital modulation techniques (BPSK, DPSK, QPSK, and QAM) based wireless communication system on the audio signal transmission through the additive Gaussian Noise (AWGN) channel is assessed on the basis of bit error rate (BER) as a function of the signal-to-noise ratio (SNR). Based on the results of this study, BPSK modulation outperforms the DPSK, QPSK, and QAM modulation strategies in the MIMO MC-CDMA VBlast based wireless communication system. The digital modulation of QPSK shows the worst performance in audio signal transmission especially in comparison to other digital modulations. It is clear from the current simulation study based on MATLAB that the V-Blast encoded $4 \times 4$ MIMO MC-CDMA wireless system with minimum mean square error (MMSE) signal detection and 1/2-rated convolution and cyclic redundancy check (CRC) channel encoding strategies show good performance utilizing BPSK digital modulation in audio signal transmission.
\end{abstract}

\section{KEYWORD}

MIMO, V-Blast, MC-CDMA, MMSE, BER, SNR, Audio Signal Transmission

\section{INTRODUCTION}

Wireless services have increased in the first place every year [1]. The current third-generation telecommunications system offers a more flexible data rate, higher capacity, and tightly integrated service. The main aim of the communication system is to transmit unit information [2]. Modulation and demodulation are the main characteristics for transmitting and receiving information on the wireless system. The modulator is therefore a key component of wireless equipment. Current mobile and wireless communication systems use the digital modulation scheme as an essential module for signal processing instead of analog modulation, which has already been used.

Digital modulation is very useful in terms of noise immunity and channel deterioration strength. In cellular wireless communication, the reuse of the same allocated frequency spectrum in different geographical areas is fascinating for the development of large-scale mobile-cellular networks. Most often, the transmission of audio signals will have to pay an enormous cost of bandwidth and a lossless reproduction is needed at the receiving end. It is therefore important to study the performance of a mobile wireless communication system with the implementation of 
International Journal of Ambient Systems and Applications (IJASA) Vol.09, No.1/2, June 2021

M-ary (MPSK, MQAM, MFSK) modulation techniques for the audio signals. These enhanced and also most successful digital modulation schemes are commonly used because of their improved spectral efficiency [3-5]. The effectiveness of the communication system depends on many factors, one of which is to evaluate the bit error rate of the technique $[6,7]$.

The goal of this article is to assess the bit error rate (BER) performance of V-Blast architecture with 4 x 4 antenna configuration for MIMO MC-CDMA wireless communication system, using MMSE signal detection scheme and 1/2-rated convolution and cyclic redundancy check (CRC) channel encoding strategies under various digital modulation schemes (BPSK, DPSK, QPSK, and 16QAM) on audio signal transmission in the presence of Additive White Gaussian Noise (AWGN) channel.

\section{RELATED WORKS}

The performance of the encrypted color image transmission in the MC-CDMA wireless communication system encoded in the D-BLAST aided LDPC is investigated in [6]. They have shown that the implementation of the QAM digital modulation technique with the deployment of the MMSE-SIC channel equalization technique provides a satisfactory result for the LDPC encoded MC-CDMA system compared to the MMSE, ZF, and ZF-SIC. Naznin et al.[7] have shown that the implementation of the MMSE-SIC signal detection technique using 4QAM digital modulation strategies verifies the effectiveness of the LDPC encoded and MP-WFRFT-based physical layer protection scheme executed by the MIMO wireless communication system to retrieve color images transmitted over noisy and Rayleigh fading channels.

Authors in [8] studied the image transmission over the MC-CDMA system and evaluated the system performance, where it was concluded that the MC-CDMA image transmission system with chaotic LMMSE equalization transmits the image efficiently when compared to the LMMSE helical interleaving system. The performance of the MIMO MC-CDMA system in QPSK, 8PSK, 8QAM, 16QAM, 32QAM, and 64QAM modulation approaches in Rayleigh fading environments is introduced in [9]. They have shown that MIMO MC-CDMA output using the QPSK modulation technique outperforms other modulation techniques with very low error probability and high gain.

A comprehensive survey on MIMO technology using the V-BLAST detection technique is presented in [10]. The performance of the MIMO MC-CDMA wireless communication system with the implementation of noise reduction and MMSE signal detection techniques for the transmission and recovery of color image signals has been studied in[11]. The authors found that Median Filter is more effective in detecting MMSE signals than in ordering statistical filters. The performance analysis of MIMO MC-CDMA uplink systems based on the V-BLAST linear zeroforce algorithm is presented in [12]. Studies have shown that the MIMO MC-CDMA system based on the linear ZF V-BLAST algorithm is capable of achieving better BER performance than the conventional MC-CDMA system by reducing the number of transmitting antennas or increasing the number of receiving antennas. In [13], the performance of a V-Blast encoded MIMO MC-CDMA wireless communication system in encrypted color image transmission was investigated. According to studies, a V-Blast encoded system is very efficient and effective in retrieving encrypted color images using ZF and MMSE signal detection and 16QAM digital modulation strategies. The performance study of video transmission in the V-Blast encoded MIMO MC-CDMA system is introduced in [14]. According to the results of the study, the VBLAST encoded 2×2 MIMO MC-CDMA wireless systems with ZF signal detection and 12-rated Convolution and CRC channel coding schemes outperforms the BPSK digital modulation in video transmission. In [15] presents secure data transmission in a V-Blast encoded MIMO MCCDMA wireless communication system. According to the findings of this study, the ZF with $1 / 2-$ 
International Journal of Ambient Systems and Applications (IJASA) Vol.09, No.1/2, June 2021

rated Convolution coding scheme outperforms the MMSE with CRC coding scheme for BPSK digital modulation.

\section{COMMunication System Model}

The simulated 4 x 4 Vertical Bell Labs Layered Space-Time (V-BLAST) multi-user multichannel encoded MIMO MC-CDMA wireless communication system is shown in Figure 1. This communication system model using different digital modulation (BPSK, DPSK, QPSK, and 16QAM) and MMSE signal detection schemes. The figure shows the step-by-step operation of each functional block. On the transmitter side, the input binary data stream is generated by the recorded audio signal. The retrieved binary data stream is passed through the other required steps (encryption: Vigenere Cipher and RSA encryption, channel encoder, interleaver) as the signal has to be transmitted. Our key issue focuses on the next block of the communication system model under consideration, where it was planned to vary the different modulation schemes in order to analyze their performance. The performance and characteristics of the system are significantly dependent on the choice of the digital modulation scheme. There is no general rule for the selection of a scheme. However, one methodology is better than the other depending on the channel, the needed level of performance, and the objective hardware trade-offs [16]. The necessary data rate, latency, link budget as well as available bandwidth must also be considered. The received signal is demodulated on the receiver side and passed through the other subsequent blocks in order to reproduce the transmitted audio signal.

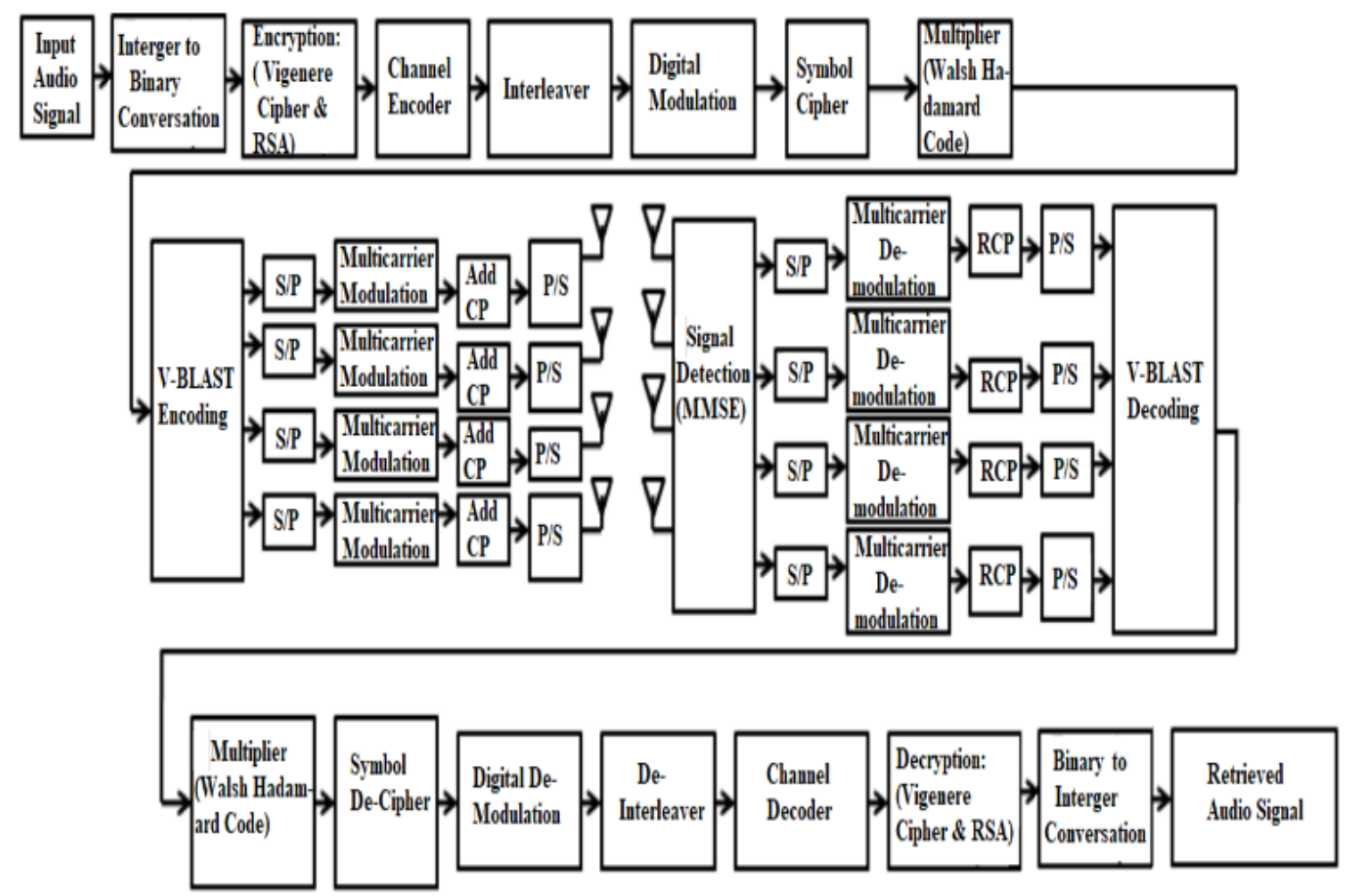

Figure 1. Block diagram of a V-Blast encoded MIMO MC-CDMA wireless communication system model. Here $\mathrm{S} / \mathrm{P}=$ serial to parallel converter; $\mathrm{P} / \mathrm{S}=$ parallel to serial converter, Add $\mathrm{CP}=$ adding cyclic prefix; $\mathrm{CP}=$ cyclic prefix. 


\section{RESUltS AND DISCUSSION}

Various modulation methodologies for wireless communication systems are evaluated utilizing voice signals as a function of the bit error rate (BER). This is the simulation work performed by Matlab taking into account the following simulation parameters as shown in Table 1. The work deals with the audio signal that is transmitted through the AWGN channel, where fading channels such as Rayleigh fading and Ricean fading channel are not considered.

Figure 2 shows the system performance under different digital modulation schemes (BPSK, DPSK, QPSK, and 16 QAM). The Bit Error Rate (BER) is significantly reduced with respect to the signal-to-noise ratio (SNR). It is clear from this figure that the performance of BPSK is better than that of others. However, DPSK and 16QAM have a nearly smaller result, while QPSK has a higher bit error rate (BER). A higher BER value can be found in QPSK at $S N R=1 \mathrm{~dB}$ than in other modulation techniques.

Table 1. Summary of the simulation model parameters.

\begin{tabular}{|l|l|}
\hline Parameters & Values \\
\hline Data Types & Audio Signal \\
\hline Input Audio size & $1,45,408$ bytes \\
\hline Channel Coding & $1 / 2$-rated Convolution and CRC Channel encoding \\
\hline Modulation & BPSK,DPSK,QPSK,16-QAM \\
\hline Cryptographic algorithm & Vigenere Cipher and RSA \\
\hline Antenna configuration & $4 \times 4$ \\
\hline Channel & AWGN \\
\hline Signal to noise ratio, SNR & 0 to 40 dB \\
\hline Spreading Code & Walsh Hadamard \\
\hline Signal detection & MMSE \\
\hline
\end{tabular}

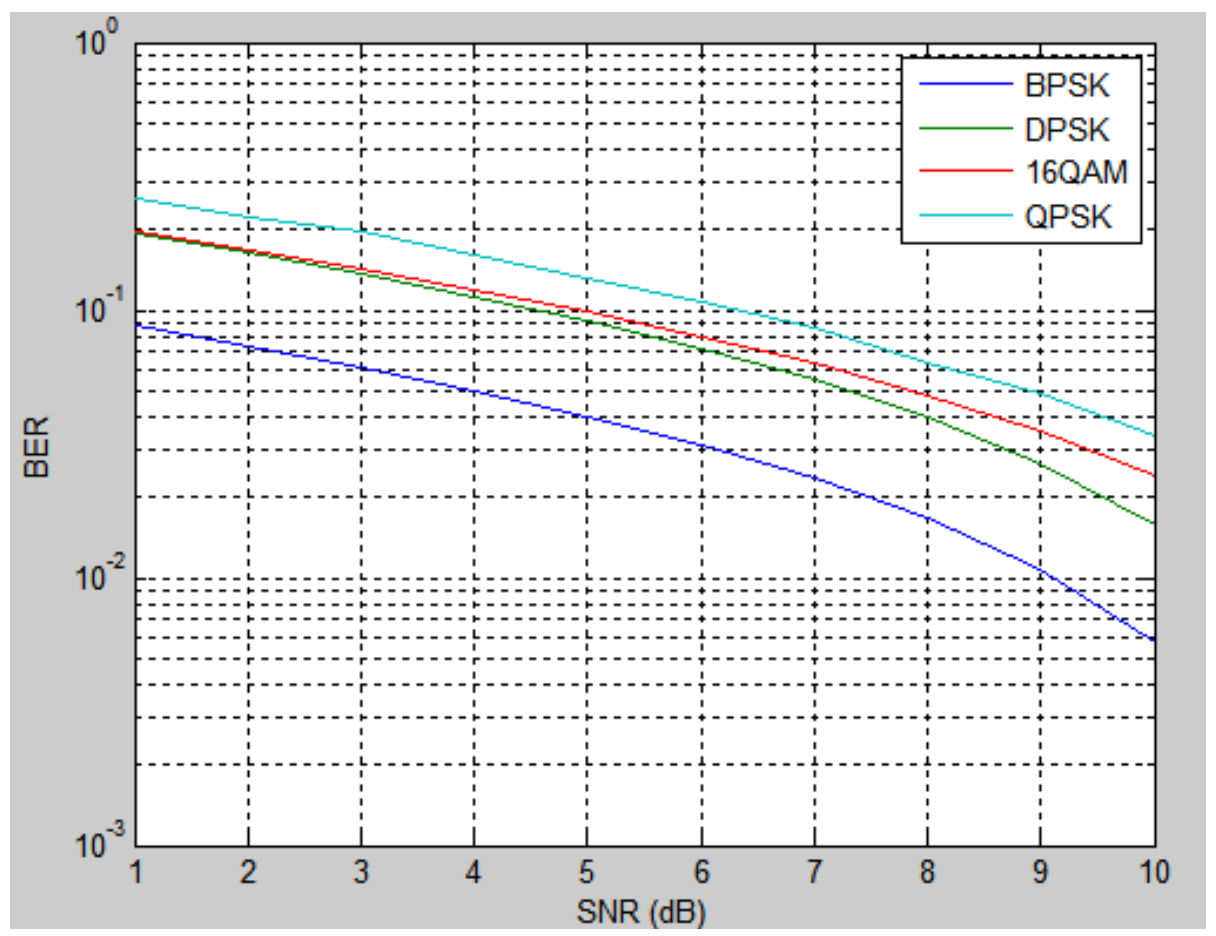

Figure 2. System performance under different modulation schemes (BPSK, DPSK, QPSK, and 16 QAM) 
International Journal of Ambient Systems and Applications (IJASA) Vol.09, No.1/2, June 2021

The BER values of the system at a different SNR for different modulation schemes of the audio signal are summarized in Table 2. From the table, it is clearly observed that for different SNR values $(\mathrm{SNR}=1-10 \mathrm{~dB})$, BPSK shows better BER performance than that of DPSK, 16QAM, and QPSK. These simulation results show that the BPSK modulated V-BLAST MIMO MC-CDMA system with the use of convolution and CRC channel encoding and the MMSE signal detection technique outperforms the transmission of audio data while the QPSK modulated system shows the worst performance.

Table 2. BER values of the V-BLAST encoded MIMO MC-CDMA system at a different SNR for various modulation schemes.

\begin{tabular}{|c|c|c|c|c|}
\hline \multirow{2}{*}{ SNR $(\mathrm{dB})$} & \multicolumn{4}{|c|}{ BER values for different modulations } \\
\cline { 2 - 5 } & BPSK & DPSK & 16 QAM & QPSK \\
\hline 1 & 0.087 & 0.1932 & 0.1962 & 0.2612 \\
\hline 2 & 0.0735 & 0.1637 & 0.1682 & 0.2242 \\
\hline 3 & 0.0611 & 0.137 & 0.1426 & 0.1965 \\
\hline 4 & 0.05 & 0.1128 & 0.1194 & 0.1598 \\
\hline 5 & 0.0401 & 0.0912 & 0.0985 & 0.1322 \\
\hline 6 & 0.0312 & 0.0718 & 0.0797 & 0.1073 \\
\hline 7 & 0.0234 & 0.0547 & 0.063 & 0.0852 \\
\hline 8 & 0.0166 & 0.0397 & 0.0482 & 0.0633 \\
\hline 9 & 0.0107 & 0.0267 & 0.0353 & 0.0485 \\
\hline 10 & 0.0057 & 0.0156 & 0.0241 & 0.0336 \\
\hline
\end{tabular}

\section{CONClusion}

In this paper, the bit error rate (BER) performance of V-Blast encoded 4 x 4 MIMO MC-CDMA wireless communication system communication using MMSE signal detection scheme and $1 / 2$ rated convolution and cyclic redundancy check (CRC) channel encoding strategies under various digital modulation schemes (BPSK, DPSK, QPSK, and 16QAM) on audio signal transmission in the presence of Additive White Gaussian Noise (AWGN) channel is studied. Based on the results of the present study, it is seen that BPSK modulation shows the finest performance compared to other digital modulation schemes (DPSK, QPSK, and 16QAM). It can therefore be concluded that a lossless reproduction of the audio signal can be achieved at the end of the receiver of the projected V-Blast encoded $4 \times 4$ MIMO MC-CDMA wireless communication system with the deployment of MMSE signal detection scheme and 1/2-rated convolution and cyclic redundancy check (CRC) channel encoding strategies under BPSK digital modulation scheme.

\section{REFERENCES}

[1] T. S. Rappaport, "Wireless Communications Principles \& Practice”, IEEE Press, New York, Prentice Hall, pp. 169-177 (1996).

[2] Md. Golam Rashed, M. HasnatKabir, Sk. EnayetUllah andRubaiyat Yasmin, "Performance evaluation of CRC Interleaved QPSK based Wireless Communication System for Color Image Transmission." Journal of Bangladesh Electronics Society (BES), Dhaka, Bangladesh. (December 2009).

[3] Hongzhi Zhao, Yi Gong, Yong Liang Guan, and YouxiTang, "Performance Analysis of MPSK/MQAM Modulated Orthogonal Space-Time Block Codes in Keyhole Channels" IEEE Transactions on Vehicular Technology, Vol. 58, No. 2, (February 2009). 
International Journal of Ambient Systems and Applications (IJASA) Vol.09, No.1/2, June 2021

[4] M. A. Razzak, F. Ndiaye, O. Khayam, A. Jabbar, "On the Performance of M-ary Modulation Schemes for Efficient Communication Systems "10th international conference on Computer and information technology, (2007).

[5] M. Riaz Ahmed, Md.Rumen Ahmed, Md.Ruhul Amin Robin, Md. Asaduzzaman, Md. MahbubHossain, Md. Abdul Awal, "Performance Analysis of Different M-Ary Modulation Techniques in Fading Channels using Different Diversity", Journal of Theoretical and Applied Information Technology, Vol. 15. No.1, pp 23-28, (May 2010).

[6] TonusreeSaha, Md. SarwarHosain and Shafi Ahmed Istiaq, Advances in Networks, 4(2), pp. 13-20 (2016).

[7] Laila Naznin, Mohammad Reaz Hossain \& Shaikh EnayetUllah, Global Journal of Computer Science and Technology: AHardware\& Computation, Vol.17, Issue 1, Version 1.0, pp. 27-38,

(2017).

[8] ShikhaJindalandDiwakar Agarwal, Proceedings of the International Conference on Advances in Computing,Communications and Informatics (ICACCI), pp. 1331-1347 (2014).

[9] Mr. Atul Singh Kushwah and Mr. Mayank Mittal, International Journal of Engineering Research \& Technology (IJERT), Vol. 3, Issue 1, pp. 2484-2490 (2014).

[10] Sudhanshu Kumar Chourasia and Rashmi Pandey, International Journal of Computer Applications (0975 - 8887) Vol. 98, no.5, pp. 38-42 (2014).

[11] Sharmin Sultana, AurangzibMdAbdur Rahman and Foez Ahmed, Journal of Multidisciplinary Engineering Science and Technology (JMEST), Vol. 3, Issue 3, pp. 4386-4391 (2016).

[12] Wei Yang, Science in China Series F Information Sciences, Vol. 51, no. 9, pp. 1305-1318

(2008)

[13] Md. Sofiqul Islam, Md. Firoz Ahmed, A. Z. M. Touhidul Islam, "Performance Analysis of V-Blast Encoded MIMO MC-CDMA Wireless Communication System in Encrypted Color Image Transmission, "International Journal of Recent Engineering Science (IJRES), Volume 7 Issue 3,(2020).

[14] Md. Juwel Sheikh, Md. Firoz Ahmed, A. Z. M. Touhidul Islam, "Video transmission in the V-Blast encoded MIMO MC-CDMA system: performance study, "International Journal of Ambient Systems and Applications (IJASA) Vol.8, No.1/2, (2020).

[15] M.Haque, M.F.Sharmin, S.E. Ullah, "Secured data transmission in a V-Blast encoded MIMO MCCDMA wireless communication system," International Journal of Information and Network Security (IJINS) 2(3), (2013).

[16] G. Smithson, "Introduction to digital modulation schemes", IEE Digest / Volume 1998 / Issue 240, doi: 10.1049/ic: 19980230 